\title{
Computing the Loewner driving process of random curves in the half plane
}

\author{
Tom Kennedy \\ Department of Mathematics \\ University of Arizona \\ Tucson, AZ 85721 \\ email: tgk@math.arizona.edu
}

October 29, 2018

\begin{abstract}
We simulate several models of random curves in the half plane and numerically compute the stochastic driving processes that produce the curves through the Loewner equation. Our models include models whose scaling limit is the Schramm-Loewner evolution (SLE) and models for which it is not. We study several tests of whether the driving process is Brownian motion, as it is for SLE. We find that testing only the normality of the process at a fixed time is not effective at determining if the random curves are an SLE. Tests that involve the independence of the increments of Brownian motion are much more effective. We also study the zipper algorithm for numerically computing the driving function of a simple curve. We give an implementation of this algorithm which runs in a time $O\left(N^{1.35}\right)$ rather than the usual $O\left(N^{2}\right)$, where $N$ is the number of points on the curve.
\end{abstract}




\section{Introduction}

The Loewner equation provides a means for encoding curves in the upper half plane that do not intersect themselves by a real-valued function. Let $\gamma(t)$ be such a simple curve with $0 \leq t<\infty$. Let $\mathbb{H}$ denote the upper half of the complex plane, and let $\gamma[0, t]$ denote the image of $\gamma$ up to time $t$. Then $\mathbb{H} \backslash \gamma[0, t]$ is a simply connected domain. So there is a conformal map $g_{t}$ from this domain to $\mathbb{H}$. If the curve is suitably parametrized and $g_{t}$ is suitably normalized, then $g_{t}$ satisfies the differential equation

$$
\frac{\partial g_{t}(z)}{\partial t}=\frac{2}{g_{t}(z)-U_{t}}, \quad g_{0}(z)=z
$$

for some real valued function $U_{t}$ on $[0, \infty)$. The function $U_{t}$ is often called the driving function.

If our simple curve in the half plane is random, then the driving function $U_{t}$ is a stochastic process. Schramm discovered that if the scaling limit of a two-dimensional model is conformally invariant and satisfies a certain Markov property, then this stochastic driving process must be a Brownian motion with mean zero [14]. The only thing that is not determined is the variance. Schramm named this process stochastic Loewner evolution or SLE; it is now often referred to as Schramm-Loewner evolution.

Many critical two-dimensional models from statistical mechanics and probability satisfy these properties or are believed to satisfy them, and so should be SLE for some $\kappa$ (the parameter that determines the variance). These include the loop-erased random walk [11, 19], the selfavoiding walk [10], interfaces in the critical Ising model [16], the Gaussian free field [17], critical percolation [15, 5], and uniform spanning trees [11]. More recent work has considered whether other models have random curves that are described by SLE. The possibility that domain walls in spin glass ground states are SLE curves was studied numerically both by Amoruso, Hartman, Hastings, and Moore [1] and by Bernard, Le Doussal, and Middleton [4]. Bernard, Boffetta, Celani and Falkovich considered simulations of certain isolines in two-dimensional turbulence [2] and surface quasi-geostrophic turbulence [3].

In this paper we consider models which are definitely not SLE. They are based on well known lattice models - the loop-erased random walk (LERW), the self-avoiding walk (SAW) and the critical percolation exploration process. We distort these models by shrinking the random curves slightly in the vertical direction but not in the horizontal direction. In other words we apply a non-conformal transformation to the curves. Without distortion these models are all proven or conjectured to have a scaling limit given by SLE.

One way to test if a model of random curves is SLE is to compute its stochastic driving process and see if it is Brownian motion. In this paper we simulate these distorted models, numerically compute their stochastic driving process, and then test if they are Brownian motions. We also do this for the models without distortion. Our goal is to see how well one can determine whether or not a model is SLE by studying this stochastic driving process and to compare various methods for testing if the stochastic driving process is a Brownian motion.

Another goal of this paper is to study the algorithm for computing the driving function of 
a given curve. The standard implementation of the "zipper algorithm" for doing this requires a time $O\left(N^{2}\right)$ where $N$ is the number of points on the curve. We present an implementation that runs in a time $O\left(N^{p}\right)$ with $p$ approximately 1.35. This implementation uses the same idea used in [6] to simulate SLE curves quickly. We also study the difference in the driving function found using "tilted slits" versus "vertical slits" and the effect of the number of points used on the curve to compute the driving function.

\section{Distorted models}

We study the stochastic driving function of three models. We refer to them as distorted models. For $\lambda>0$ we define a non-conformal map on the upper half plane by $\phi(x, y)=(x, \lambda y)$. Given a model that produces random curves $\gamma$ in the upper half plane, we consider the random curves $\phi \circ \gamma$. In other words, we stretch the curve by a factor of $\lambda$ in the vertical direction, but do not stretch it in the horizontal direction.

We apply this distortion to the loop-erased random walk, the self-avoiding walk and percolation interfaces. For all three models we consider the chordal version of the model in which the random curve lies in the upper half plane and goes from the origin to $\infty$. For the LERW this means we take a half plane excursion and loop erase it. For the SAW this means we use the uniform probability measure on nearest neighbor walks with a fixed number of steps which begin at the origin and lie in the upper half plane. For percolation we consider site percolation on the triangular lattice in the upper half plane with boundary conditions which force an interface to start at the origin. Details of the definitions of these models and the parameters used in the simulations may be found in the appendix.

There is no simple relation between the driving function for the curve $\gamma$ and the driving function for the distorted curve $\phi \circ \gamma$. We study the driving function for the distorted model as follows. We generate samples of the LERW, SAW or percolation interface and then apply the distortion map $\phi$. Then we compute the driving function of the distorted curve. The result is a collection of samples of the stochastic driving process of the distorted model. We then do various statistical tests to see if this process is a Brownian motion.

We denote the driving process by $U_{t}$. All the models are invariant under reflections about the vertical axis. Hence $E\left[U_{t}\right]=0$. We begin by plotting the variance $E\left[U_{t}^{2}\right]$ as a function of $t$. We should emphasize that in the scaling limit, all the models have a scaling property which implies that $E\left[U_{t}^{2}\right]$ is a linear function of $t$, even if the scaling limit is not an $S L E$. So this does not test whether $U_{t}$ is a Brownian motion. It only provides an estimate of $\kappa$ where $\kappa$ is the slope of the function $t \rightarrow E\left[U_{t}^{2}\right]$.

The first statistical test is to see if the distribution of an individual $U_{t}$ is normal. We use the Kolmogorov-Smirnov test. This test is based on the fact that for a continuous random variable $Y$, if $F$ is the cumulative distribution of $Y$, then $F(Y)$ is uniformly distributed on $[0,1]$. Let $Y_{1}, Y_{2}, \cdots, Y_{N}$ be $N$ observations of the random variable $Y$, and let $Y_{(1)}<Y_{(2)}<\cdots Y_{(N)}$ be 
these numbers arranged in increasing order. Then the statistic is

$$
D=\max _{1 \leq k \leq N}\left|F\left(Y_{(k)}\right)-\frac{k+\frac{1}{2}}{N}\right|+\frac{1}{2 N}
$$

(This formula may appear different from that found in most statistics texts, but it is in fact the same.) Under the null hypothesis that $Y$ has the distribution $F$, the limiting distribution of $\sqrt{N} D$ as $N \rightarrow \infty$ is known. For example, $P(\sqrt{N} D>1.36)$ is approximately $5 \%$. So if we compute this statistic for an individual $U_{t}$ with $F$ equal to the cumulative distribution for a normal random variable with mean zero and variance $\kappa t$, and find that the value of $\sqrt{N} D$ is large (say larger than 1.36), then we conclude that the distribution of $U_{t}$ is not this normal distribution. We perform this Kolmogorov-Smirnov test for two values of the time, $T$ and $T / 2$. The value of $T$ as well as other parameters used in the simulations are given in the appendix. The results are shown in the various tables in the columns labeled $D(T / 2)$ and $D(T)$.

Our next test involves the independence of increments of Brownian motion. Let

$$
X_{1}=U_{T / 2}, \quad X_{2}=U_{T}-U_{T / 2}
$$

If $U_{t}$ is a Brownian motion, then $X_{1}$ and $X_{2}$ are independent and have mean zero. So their product $X_{1} X_{2}$ has mean zero. We test the hypothesis that its mean is zero. If $U_{t}$ is a Brownian motion, then the variance of $X_{1} X_{2}$ can be found. Denote it by $\sigma^{2}$. The statistic we use, $Z$, is simply the sample mean for $X_{1} X_{2}$ divided by $\sigma / \sqrt{N}$. If $N$ is large and $U_{t}$ is a Brownian motion, then the distribution of $Z$ is close to that of the standard normal. This test is shown in the tables in the column labeled $Z$.

For our last three tests we let $0<t_{1}<t_{2}<\cdots<t_{n}=T$. We use equally spaced $t_{i}$ in our tests, but one could consider non-uniform choices. If $U_{t}$ is a Brownian motion, then the increments

$$
X_{j}=U_{t_{j}}-U_{t_{j-1}}
$$

are independent and each is normal with mean zero and variance $\kappa\left(t_{j}-t_{j-1}\right)$. We test this joint distribution with a $\chi^{2}$ goodness-of-fit test. The idea is to divide the possible values of $\left(X_{1}, X_{2}, \cdots, X_{n}\right)$ into $m$ cells and count the number of samples that fall into each cell. Under the hypothesis that the $X_{j}$ are independent and normally distributed, we can compute the expected number of samples that fall into each cell. Let $O_{j}$ be the number of samples in cell $j$, and $E_{j}$ the expected value of this random variable under the hypothesis that $U_{t}$ is Brownian motion. The test is then based on the statistic:

$$
\chi^{2}=\sum_{j=1}^{m} \frac{\left(O_{j}-E_{j}\right)^{2}}{E_{j}}
$$

Under the hypothesis that $U_{t}$ is a Brownian motion, if $m$ is large the distribution of this statistic is approximately the $\chi^{2}$ distribution with $m-1$ degrees of freedom.

We consider three choices of the cells. In the first choice we let $n=10$, and use only the signs of the $X_{j}$ to define the cells. Thus there are $2^{10}=1024$ cells. We denote the statistic in this case 


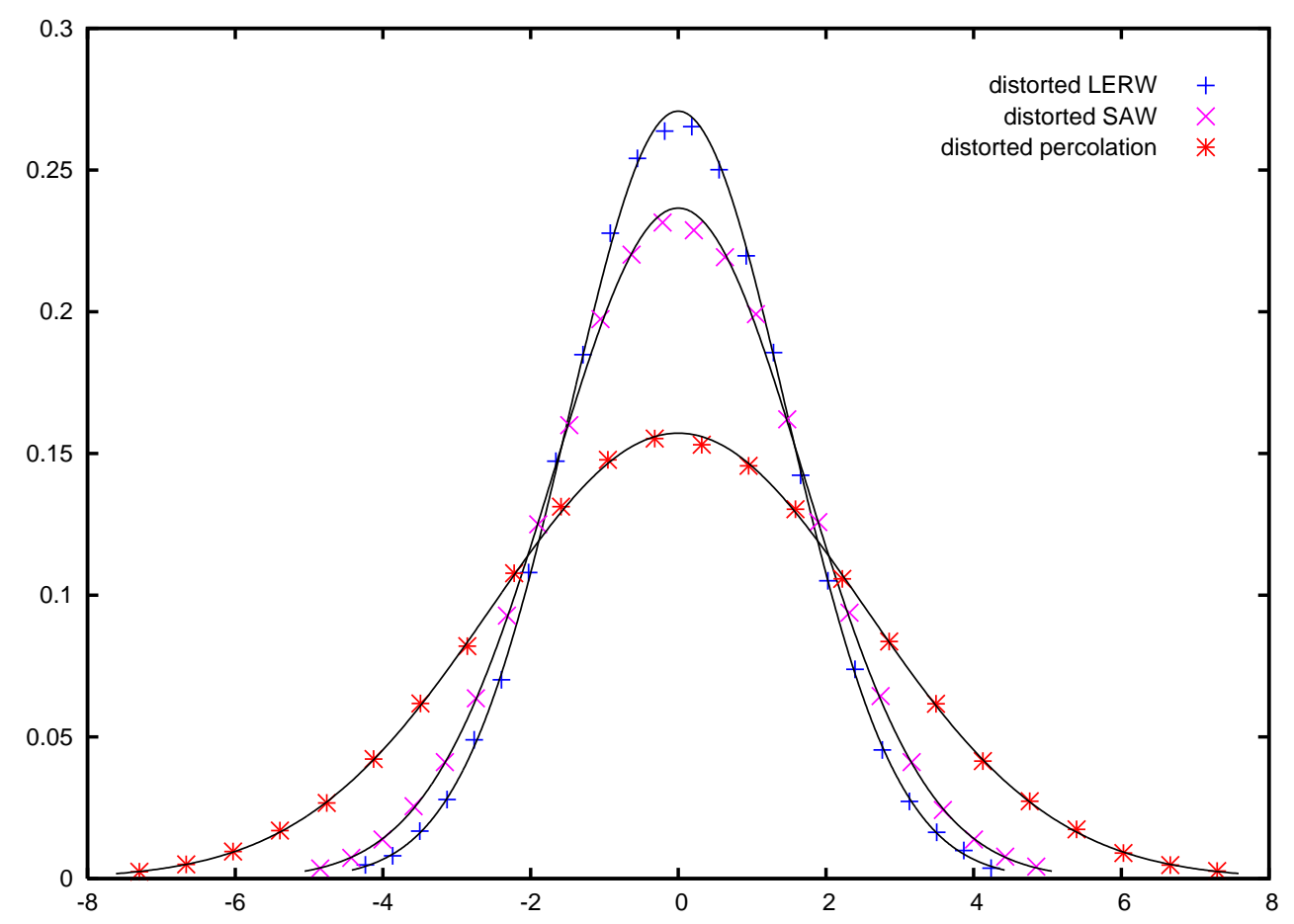

Figure 1: The points are a histogram for the density of $U_{T} / \sqrt{T}$ for the LERW, SAW and percolation with distortion $\lambda=0.95$. The curves are the density of a normal distribution with variance $\kappa$ where $\kappa$ is determined from the least squares fit.

by $\chi_{a}^{2}$. The second choice of cells is similar - we use only the signs of the increments but with $n=5$. So there are $2^{5}=32$ cells. This statistic is denoted by $\chi_{b}^{2}$. The third choice uses just two increments. For each of the increments $X_{1}$ and $X_{2}$ we look at which quartile it falls in. We let $q$ be the constant such that under the hypothesis that $U_{t}$ is Brownian motion, $P\left(X_{i}>q\right)=1 / 4$, and then divide the possible values of each $X_{i}$ into the intervals $(-\infty,-q],[-q, 0],[0, q],[q, \infty)$. Thus there are 16 cells. This final statistic is denoted by $\chi_{c}^{2}$. Note that the statistics $\chi_{a}^{2}$ and $\chi_{b}^{2}$ have the advantage that they do not involve the value of $\kappa$. For $\chi_{c}^{2}$ we need a value for $\kappa$ to compute $q$.

We study three values of the distortion parameter, $\lambda=0.9,0.95$ and 1 , for each of the three lattice models. Thus there are a total of nine models considered. (We have run the same simulations for $\lambda=1.05$ and 1.1, but do not present these results. They are consistent with the results that we do present.) We generate $10^{5}$ samples for each of the nine cases, compute the driving function $U_{t}$ for each sample, and record the value of $U_{t}$ at ten equally spaced times, $t=T / 10,2 T / 10, \cdots, T$. We compute the variance of $U_{t}$ at each of the ten times and then do a least squares fit to estimate $\kappa$, the slope of $t \rightarrow E\left[U_{t}^{2}\right]$. The results are shown in table 1.

For each of the three models we present the results of our statistical tests in a table. 


\begin{tabular}{|c|c|c|}
\hline Model & $\lambda$ & $\kappa$ \\
\hline LERW & 0.90 & $2.3315 \pm 0.0106$ \\
LERW & 0.95 & $2.1709 \pm 0.0094$ \\
LERW & 1.00 & $2.0008 \pm 0.0093$ \\
\hline SAW & 0.90 & $3.0744 \pm 0.0138$ \\
SAW & 0.95 & $2.8414 \pm 0.0108$ \\
SAW & 1.00 & $2.6686 \pm 0.0132$ \\
\hline percolation & 0.90 & $6.9446 \pm 0.0287$ \\
percolation & 0.95 & $6.4422 \pm 0.0311$ \\
percolation & 1.00 & $6.0404 \pm 0.0265$ \\
\hline
\end{tabular}

Table 1: The estimate of $\kappa$ using a weighted least squares fit for $E\left[U_{t}^{2}\right]$ as a function of $t$. The error bars are two standard deviations.

We perform the statistical tests for the first $N$ samples of the $100 K$ samples, using $N=$ $5 K, 10 K, 20 K, 50 K$ and $100 K$. In the tables we do not give the value of the statistic, but rather the corresponding "p-value." The p-value is defined as follows. Consider a one-sided statistic such as the Kolmogorov-Smirnov $D$ statistic. Suppose that in our test the value of the statistic is $x$. Then the p-value is the probability $P(D>x)$ under the null hypothesis. For a two-sided statistic such as $Z$ the definition is modified in the obvious way. A small p-value (less than a few percent) indicates that the value of the corresponding statistic is very unlikely under the hypothesis that $U_{t}$ is Brownian motion, and so we should reject the hypothesis that $U_{t}$ is Brownian motion.

These nine tables contain a lot of numbers, but they clearly show the following. For $\lambda=1$ the p-values in the table for all three lattice models are not small, and so our tests do not indicate that we should reject the hypothesis that $U_{t}$ is a Brownian motion. This is as it should be. When $\lambda=1$ the models should have a scaling limit given by $S L E$, and so $U_{t}$ should indeed be Brownian motion.

For the models that are not SLE, $\lambda=0.9,0.95$, we first consider the two KolmogorovSmirnov tests that $U_{t}$ is normal. The corresponding p-values are not typically small, and this test is completely ineffective at indicating that $U_{t}$ is not Brownian motion, even when we use $10^{5}$ samples. In figure 1 the data points are histograms for the density function of $U_{T} / \sqrt{T}$ for the LERW, SAW and percolation with $\lambda=0.95$. The curves are the density functions for the normal distribution with variance $\kappa$ where $\kappa$ is determined from our least squares fit. As the Kolmogorov-Smirnov test showed, the data points are fit very well by the normal curves. (For $\lambda \neq 1$, it is easy to show that the distorted model is not SLE, and so $U_{t}$ is not a Brownian motion. However, this does not rule out the possibility that the $U_{t}$ are normal even for the distorted models.)

The other four tests involve the independence of the increments. The test based on the mean of the product of two independent increments sometimes indicates correctly that $U_{t}$ is 


\begin{tabular}{|c|c|c|c|c|c|c|c|}
\hline$\lambda$ & $\mathrm{N}$ & $D(T / 2)$ & $D(T)$ & $Z$ & $\chi_{a}^{2}$ & $\chi_{b}^{2}$ & $\chi_{c}^{2}$ \\
\hline \multirow{5}{*}{0.90} & 5,000 & 0.018695 & 0.223875 & 0.012146 & 0.306555 & 0.654153 & 0.139523 \\
& 10,000 & 0.605601 & 0.562645 & 0.012238 & 0.000614 & 0.000666 & 0.352671 \\
& 20,000 & 0.578409 & 0.860558 & 0.029349 & 0.000000 & 0.000041 & 0.820988 \\
& 50,000 & 0.213470 & 0.308500 & 0.078267 & 0.000000 & 0.000000 & 0.000120 \\
& 100,000 & 0.222762 & 0.253184 & 0.003394 & 0.000000 & 0.000000 & 0.000000 \\
\hline \hline \multirow{5}{*}{0.95} & 5,000 & 0.689088 & 0.567792 & 0.021239 & 0.913735 & 0.598610 & 0.766046 \\
& 10,000 & 0.562020 & 0.898586 & 0.055965 & 0.423453 & 0.350098 & 0.593728 \\
& 20,000 & 0.648585 & 0.638202 & 0.064346 & 0.793825 & 0.318862 & 0.865687 \\
& 50,000 & 0.486077 & 0.178479 & 0.010384 & 0.078510 & 0.000173 & 0.850495 \\
& 100,000 & 0.234619 & 0.004251 & 0.000812 & 0.000080 & 0.000000 & 0.501958 \\
\hline \hline \multirow{5}{*}{1.00} & 5,000 & 0.322835 & 0.909183 & 0.607075 & 0.599810 & 0.281570 & 0.332253 \\
& 10,000 & 0.902220 & 0.499358 & 0.274931 & 0.118588 & 0.593618 & 0.820108 \\
& 20,000 & 0.763553 & 0.856747 & 0.240621 & 0.167155 & 0.227972 & 0.322061 \\
& 50,000 & 0.840997 & 0.746111 & 0.376101 & 0.695880 & 0.735182 & 0.702162 \\
& 100,000 & 0.949877 & 0.934915 & 0.448990 & 0.305246 & 0.257668 & 0.509009 \\
\hline
\end{tabular}

Table 2: The distorted LERW. $\lambda$ is the amount of distortion, with $\lambda=1$ being no distortion. $N$ is the number of samples used. The other six columns give the p-value of six different statistics used to test if the driving process is Brownian motion. See the text for details.

not Brownian motion, but it is not very powerful. By contrast $\chi_{a}^{2}$ and $\chi_{b}^{2}$ are quite effective at correctly indicating when $U_{t}$ is not a Brownian motion. For $\lambda=0.9$ these tests correctly indicate $U_{t}$ is not a Brownian motion with sample sizes on the order of ten to twenty thousand. For $\lambda=0.95$ these tests need on the order of a hundred thousand samples, but they are the only tests to correctly indicate that $U_{t}$ is not a Brownian motion for this amount of distortion. The final statistic $\chi_{c}^{2}$ is only sometimes effective.

\section{A faster zipper}

We briefly describe the standard method for computing the driving function of a simple curve $\gamma$. Let $g_{s}$ be the conformal map which takes the half plane minus $\gamma[0, s]$ onto the half plane, normalized so that for large $z$

$$
g_{s}(z)=z+\frac{2 t}{z}+O\left(\frac{1}{z^{2}}\right),
$$

The coefficient $2 t$ depends on $s$ and is the half-plane capacity of $\gamma[0, s]$. The value of the driving function at $t$ is $U_{t}=g_{s}(\gamma(s))$. Thus computing the driving function essentially reduces to computing this uniformizing conformal map. We will describe the "zipper algorithm" for doing this [8, 13]. Another approach to computing the driving function may be found in [18]. 


\begin{tabular}{|c|c|c|c|c|c|c|c|}
\hline$\lambda$ & $\mathrm{N}$ & $D(T / 2)$ & $D(T)$ & $Z$ & $\chi_{a}^{2}$ & $\chi_{b}^{2}$ & $\chi_{c}^{2}$ \\
\hline \multirow{5}{*}{0.90} & 5,000 & 0.076330 & 0.174654 & 0.538122 & 0.218454 & 0.170313 & 0.903646 \\
& 10,000 & 0.471555 & 0.343790 & 0.824175 & 0.100552 & 0.019827 & 0.803437 \\
& 20,000 & 0.246740 & 0.321011 & 0.905811 & 0.003206 & 0.000008 & 0.464170 \\
& 50,000 & 0.165784 & 0.312940 & 0.013581 & 0.000000 & 0.000000 & 0.004110 \\
& 100,000 & 0.251134 & 0.200176 & 0.000124 & 0.000000 & 0.000000 & 0.000000 \\
\hline \hline \multirow{5}{*}{0.95} & 5,000 & 0.408348 & 0.350261 & 0.933527 & 0.027830 & 0.037820 & 0.883070 \\
& 10,000 & 0.136263 & 0.888704 & 0.681952 & 0.775130 & 0.704539 & 0.931479 \\
& 20,000 & 0.805490 & 0.663008 & 0.983501 & 0.682962 & 0.797951 & 0.104660 \\
& 50,000 & 0.483299 & 0.323554 & 0.882502 & 0.162307 & 0.030086 & 0.959757 \\
& 100,000 & 0.313017 & 0.158268 & 0.794145 & 0.000018 & 0.009703 & 0.534966 \\
\hline \hline \multirow{5}{*}{1.00} & 5,000 & 0.456948 & 0.371554 & 0.636449 & 0.618423 & 0.126116 & 0.424437 \\
& 10,000 & 0.557806 & 0.267715 & 0.821561 & 0.200096 & 0.145614 & 0.609878 \\
& 20,000 & 0.933089 & 0.552806 & 0.514615 & 0.793825 & 0.253454 & 0.370379 \\
& 50,000 & 0.956776 & 0.852597 & 0.588566 & 0.685205 & 0.275748 & 0.161052 \\
& 100,000 & 0.501460 & 0.870474 & 0.219944 & 0.028353 & 0.079678 & 0.579479 \\
\hline
\end{tabular}

Table 3: The p-values of the distorted SAW.

We find it more convenient to work with the conformal map

$$
h_{s}(z)=g_{s}(z)-U_{s}
$$

It maps $\mathbb{H} \backslash \gamma[0, s]$ onto $\mathbb{H}$ and sends the tip $\gamma(s)$ to the origin. The value of the driving function at $s$ is minus the constant term in the Laurent expansion of $h_{s}$ about $\infty$. From now on we work with this normalization for our conformal maps.

Let $z_{0}, z_{1}, \cdots, z_{n}$ be points along the curve with $z_{0}=0$. In our applications these are lattice sites. The zipper algorithm finds a sequence of conformal maps $h_{i}, i=1,2, \cdots, n$ such that $h_{k} \circ h_{k-1} \circ \cdots \circ h_{1}$ approximates the conformal map for the curve up to site $z_{k}$. Suppose that the conformal maps $h_{1}, h_{2}, \cdots, h_{k}$ have been defined so that $h_{k} \circ h_{k-1} \circ \cdots \circ h_{1}$ sends $\mathbb{H} \backslash \gamma$ to $\mathbb{H}$ where $\gamma$ is some curve that passes through $z_{0}, z_{1}, \cdots z_{k}$. In particular $z_{k}$ is mapped to the origin. Let

$$
w_{k+1}=h_{k} \circ h_{k-1} \circ \cdots \circ h_{1}\left(z_{k+1}\right)
$$

Then $w_{k+1}$ is close to the origin. We define $h_{k+1}$ to be a conformal map with the appropriate normalizations that sends $\mathbb{H} \backslash \gamma_{k+1}$ to $\mathbb{H}$ where $\gamma_{k+1}$ is a short simple curve that ends at $w_{k+1}$. The key idea is to choose this curve so that $h_{k+1}$ is explicitly known. The two choices we will use are "tilted slits" and "vertical slits."

Let $2 \Delta t_{i}$ be the capacity of the map $h_{i}$, and $\Delta U_{i}$ the final value of the driving function for $h_{i}$. So

$$
h_{i}(z)=z-\Delta U_{i}+\frac{2 \Delta t_{i}}{z}+O\left(\frac{1}{z^{2}}\right)
$$




\begin{tabular}{|c|c|c|c|c|c|c|c|}
\hline$\lambda$ & $\mathrm{N}$ & $D(T / 2)$ & $D(T)$ & $Z$ & $\chi_{a}^{2}$ & $\chi_{b}^{2}$ & $\chi_{c}^{2}$ \\
\hline \multirow{5}{*}{0.90} & 5,000 & 0.268811 & 0.707407 & 0.035275 & 0.463308 & 0.440588 & 0.068530 \\
& 10,000 & 0.568890 & 0.306491 & 0.043429 & 0.387697 & 0.125325 & 0.286280 \\
& 20,000 & 0.113505 & 0.640373 & 0.192064 & 0.002475 & 0.000123 & 0.387076 \\
& 50,000 & 0.073017 & 0.749263 & 0.000274 & 0.000000 & 0.000000 & 0.007620 \\
& 100,000 & 0.000694 & 0.015332 & 0.000005 & 0.000000 & 0.000000 & 0.000000 \\
\hline \hline \multirow{5}{*}{0.95} & 5,000 & 0.667251 & 0.576948 & 0.881721 & 0.978563 & 0.820268 & 0.261246 \\
& 10,000 & 0.831016 & 0.921401 & 0.964028 & 0.586700 & 0.601949 & 0.198512 \\
& 20,000 & 0.827859 & 0.938272 & 0.703387 & 0.580099 & 0.123268 & 0.124509 \\
& 50,000 & 0.504299 & 0.913135 & 0.465606 & 0.033206 & 0.000071 & 0.291786 \\
& 100,000 & 0.084361 & 0.515339 & 0.259067 & 0.000000 & 0.000000 & 0.043819 \\
\hline \hline \multirow{5}{*}{1.00} & 5,000 & 0.075710 & 0.685162 & 0.242412 & 0.884633 & 0.296115 & 0.657406 \\
& 10,000 & 0.882136 & 0.304884 & 0.618193 & 0.267297 & 0.246049 & 0.766046 \\
& 20,000 & 0.271328 & 0.037469 & 0.133359 & 0.088622 & 0.587819 & 0.060798 \\
& 50,000 & 0.100113 & 0.008876 & 0.184139 & 0.256426 & 0.996808 & 0.283681 \\
& 100,000 & 0.333954 & 0.059206 & 0.518117 & 0.287868 & 0.779478 & 0.149034 \\
\hline
\end{tabular}

Table 4: The p-values of the distorted percolation interface.

Then

$$
h_{k} \circ h_{k-1} \circ \cdots \circ h_{1}(z)=z-U_{t}+\frac{2 t}{z}+O\left(\frac{1}{z^{2}}\right)
$$

where

$$
\begin{gathered}
t=\sum_{i=1}^{k} \Delta t_{i} \\
U_{t}=\sum_{i=1}^{k} \Delta U_{i}
\end{gathered}
$$

Thus the driving function of the curve is obtained by "adding up" the driving functions of the elementary conformal maps $h_{i}$.

We now consider the two particular types of maps we use for $h_{k+1}$. For tilted slits, $\gamma_{k+1}$ is the line segment from the origin to $w_{k+1}$. There is no explicit formula for $h_{k+1}$ in this case, but there is a formula for its inverse:

$$
h_{k+1}^{-1}(z)=\left(z+x_{l}\right)^{1-\alpha}\left(z-x_{r}\right)^{\alpha}
$$

where $x_{l}, x_{r}>0$. It maps the half plane onto the half plane minus a line segment which starts at the origin and forms an angle $\alpha$ with the positive real axis. The interval $\left[-x_{l}, x_{r}\right]$ gets mapped onto the slit. We must choose $x_{l}$ and $x_{r}$ so that $h_{k+1}$ satisfies our normalization conditions. 
In particular, $h_{k+1}^{-1}$ must send the origin to the tip of the line segment, i.e., $w_{k+1}$. Tedious but straightforward calculation shows if we let $w_{k+1}=r \exp (i \alpha \pi)$, then

$$
x_{l}=r\left(\frac{1-\alpha}{\alpha}\right)^{\alpha}, \quad x_{r}=r\left(\frac{\alpha}{1-\alpha}\right)^{1-\alpha}
$$

The changes in the driving function are given by

$$
\Delta t=\frac{1}{4} r^{2} \alpha^{1-2 \alpha}(1-\alpha)^{2 \alpha-1}, \quad \Delta U=r(1-2 \alpha) \alpha^{-\alpha}(1-\alpha)^{-(1-\alpha)}
$$

For vertical slits we take $\gamma_{k+1}$ to be the vertical line segment from the real axis to $w_{k+1}$. Note that since this $\gamma_{k+1}$ does not start at the origin, this method does not approximate the original simple curve $\gamma$ by another simple curve. Instead, the domain of the conformal map that we construct to approximate $h_{s}$ is of the form $\mathbb{H}$ minus a set that is more complicated than a simple curve. This may make the reader nervous, but we will see in the next section that we get essentially the same driving functions using tilted slits or vertical slits. The conformal map that removes this vertical line with our normalizations is

$$
h_{k+1}(z)=i \sqrt{-(z-x)^{2}-y^{2}}
$$

where $w_{k+1}=x+i y$ and the branch cut for the square root is the negative real axis. The changes in the driving function are given by

$$
\Delta t=\frac{1}{4} y^{2}, \quad \Delta U=x
$$

A comment on terminology is in order. We use "zipper algorithm" to refer to all the various algorithms we can get from different choices of the curve $\gamma_{k+1}$. Marshall and Rohde [13] use "zipper" to refer only to the choice using tilted slits.

The number of operations needed to compute a single $w_{k+1}$ is proportional to $k$. So to compute all the points $w_{k+1}$ requires a time $O\left(N^{2}\right)$. To do better we must avoid evaluating the $k$-fold composition in (8) every time we compute a $w_{k+1}$. We begin by grouping the functions in (8) into blocks. We denote the number of functions in a block by $b$. Let

$$
H_{j}=h_{j b} \circ h_{j b-1} \circ \cdots \circ h_{(j-1) b+2} \circ h_{(j-1) b+1}
$$

If we write $k$ as $k=m b+r$ with $0 \leq r<b$, then (8) becomes

$$
w_{k+1}=h_{m b+r} \circ h_{m b+r-1} \circ \cdots \circ h_{m b+1} \circ H_{m} \circ H_{m-1} \circ \cdots \circ H_{1}\left(z_{k+1}\right)
$$

Typically, the number of compositions in (19) is smaller than the number in (8) by roughly a factor of $b$. The $h_{i}$ are relatively simple, but the composition $H_{j}$ cannot be explicitly computed. Our strategy is to approximate the $h_{i}$ by functions whose compositions can be explicitly computed to give an explicit approximation to $H_{j}$. This allows us to compute the compositions in (18) just once rather than every time we compute a $w_{k}$. 
Recall that $h_{i}$ is normalized so that $h_{i}(\infty)=\infty$ and $h_{i}^{\prime}(\infty)=1$. It maps $\mathbb{H}$ minus a simple curve which starts at the origin to $\mathbb{H}$. Let $h$ denote such a conformal map. Let $r$ be the largest distance from the origin to a point on the curve. Then $h$ is analytic on $\{z \in \mathbb{H}:|z|>r\}$. Note that $h$ is real valued on the real axis. By the Schwarz reflection principle it may be analytically continued to $\{z \in \mathbb{C}:|z|>r\}$. Moreover, it does not vanish on this domain. So if we let $f(z)=h(1 / z)$, then $f$ is analytic in $\{z \in \mathbb{C}:|z|<1 / r\}$ and $f(0)=0, f^{\prime}(0)=1$. The Laurent series of $h$ about $\infty$ is just the power series of $f$ about 0 . For large $z, h(z)$ is well approximated by a finite number of terms in this Laurent series. It will prove more convenient to work with a different series.

Define $\hat{h}(z)=1 / h(1 / z)$. Since $h(z)$ does not vanish on $\{z \in \mathbb{C}:|z|>r\}, \hat{h}(z)$ is analytic in $\{z \in \mathbb{C}:|z|<1 / r\}$. Our assumptions on $h$ imply that $\hat{h}(0)=0$ and $\hat{h}^{\prime}(0)=1$. So $\hat{h}$ has a power series of the form

$$
\hat{h}(z)=\sum_{j=1}^{\infty} a_{j} z^{j}
$$

with $a_{1}=1$. The radius of convergence of this power series is $1 / r$. Note that the coefficients of this power series are the coefficients of the Laurent series of $1 / h$.

The primary advantage of working with the power series of $\hat{h}$ is its behavior with respect to composition. It is trivial to check that

$$
\left(h_{1} \circ h_{2}\right)^{\wedge}=\hat{h_{1}} \circ \hat{h_{2}}
$$

Our approximation for $h_{i}(z)$ is to replace $\hat{h}_{i}(z)$ by the truncation of its power series at order $n$. So

$$
h_{i}(z)=\frac{1}{\hat{h}_{i}(1 / z)} \approx\left[\sum_{j=1}^{n} a_{j} z^{-j}\right]^{-1}
$$

For each $h_{i}$ we compute the power series of $\hat{h}_{i}$ to order $n$. We use them and (21) to compute the power series of $\hat{H}_{j}$ to order $n$. Let $1 / R_{j}$ be the radius of convergence for the power series of $\hat{H}_{j}$. Now consider equation (19). If $z$ is large compared to $R_{j}$, then $H_{j}(z)$ is well approximated using the power series of $\hat{H}_{j}$. We introduce a parameter $L>1$ and use this series to compute $H_{j}(z)$ whenever $|z| \geq L R_{j}$. When $|z|<L R_{j}$, we just use (18) to compute $H_{j}(z)$. The argument of $H_{j}$ is the result of applying the previous conformal maps to some $z_{k+1}$, and so is random. Thus whether or not we can approximate a particular $H_{j}$ by its series depends on the randomness and on which $w_{k+1}$ we are computing.

We need to compute $R_{j}$. Consider the images of $z_{(j-1) b}, z_{(j-1) b+1}, \cdots z_{j b-1}$ under the map $H_{j-1} \circ H_{j-2} \circ \cdots \circ H_{1}$. The domain of the conformal map $H_{j}$ is the half-plane $\mathbb{H}$ minus some curve $\Gamma_{j}$ which passes through the images of these points. The radius $R_{j}$ is the maximal distance from the origin to a point on $\Gamma_{j}$. This distance should be very close to or even equal to the maximum distance from the origin to images of $z_{(j-1) b}, z_{(j-1) b+1}, \cdots z_{j b-1}$ under $H_{j-1} \circ H_{j-2} \circ \cdots \circ H_{1}$. So we take $R_{j}$ to be the maximum of these distances. 


\begin{tabular}{|r|r|r|r|r|}
\hline $\mathrm{N}$ & time 1 & time 2 & factor & block length \\
\hline 1,000 & 0.21 & 0.43 & 0.50 & 20 \\
2,000 & 0.86 & 0.95 & 0.91 & 20 \\
5,000 & 5.44 & 3.00 & 1.81 & 20 \\
10,000 & 21.44 & 7.41 & 2.89 & 30 \\
20,000 & 85.65 & 18.31 & 4.68 & 40 \\
50,000 & 534.8 & 62.6 & 8.54 & 50 \\
100,000 & 2128 & 158 & 13.45 & 75 \\
200,000 & 8562 & 437 & 19.59 & 100 \\
500,000 & 53516 & 1674 & 31.98 & 200 \\
$1,000,000$ & 214451 & 4675 & 45.87 & 200 \\
\hline
\end{tabular}

Table 5: The time (in seconds) needed to unzip a SAW with $N$ steps without using the power series approximation is shown in the second column (time 1) The time using the power series approximation is shown in the third column (time 2). The fourth column (factor) is the ratio of these two times. The block length used is in the last column.

Our algorithm depends on three parameters. The integer $b$ is the number of functions in a block. The integer $n$ is the order at which we truncate the power series of the $\hat{H}_{j}$. The real number $L>1$ determines when we use the power series approximation for the block function.

The improvement in the speed of the zipper algorithm from using our power series approximation is shown in table 5 and figure 2. In these timing tests we use a single SAW with one million steps. We time how long it takes to unzip the first $N$ steps with and without the power series approximation. We do the computations using the power series approximation for different choices for the block length, namely $b=20,30,40,50,75,100,200,300$, and report the fastest time. The last column in the table indicates the block length that achieves the fastest time. As a rule of thumb, a good choice for the block length (at least for the SAW) is $b=\sqrt{N} / 4$. The next to last column in the table gives the factor by which the use of the power series approximation reduces the time needed for the computation. These timing tests were done on a $\mathrm{PC}$ with a $3.4 \mathrm{GHz}$ Pentium 4 processor.

Without the power series approximation the time is $O\left(N^{2}\right)$. This is seen clearly in the loglog plot in figure 2 where the data for unzipping without the power series approximation is fit quite well by a line with slope 2. The data for unzipping using the power series approximation is fit by a line with slope 1.35. This indicates that the time required when the power series are used is approximately $O\left(N^{1.35}\right)$. 


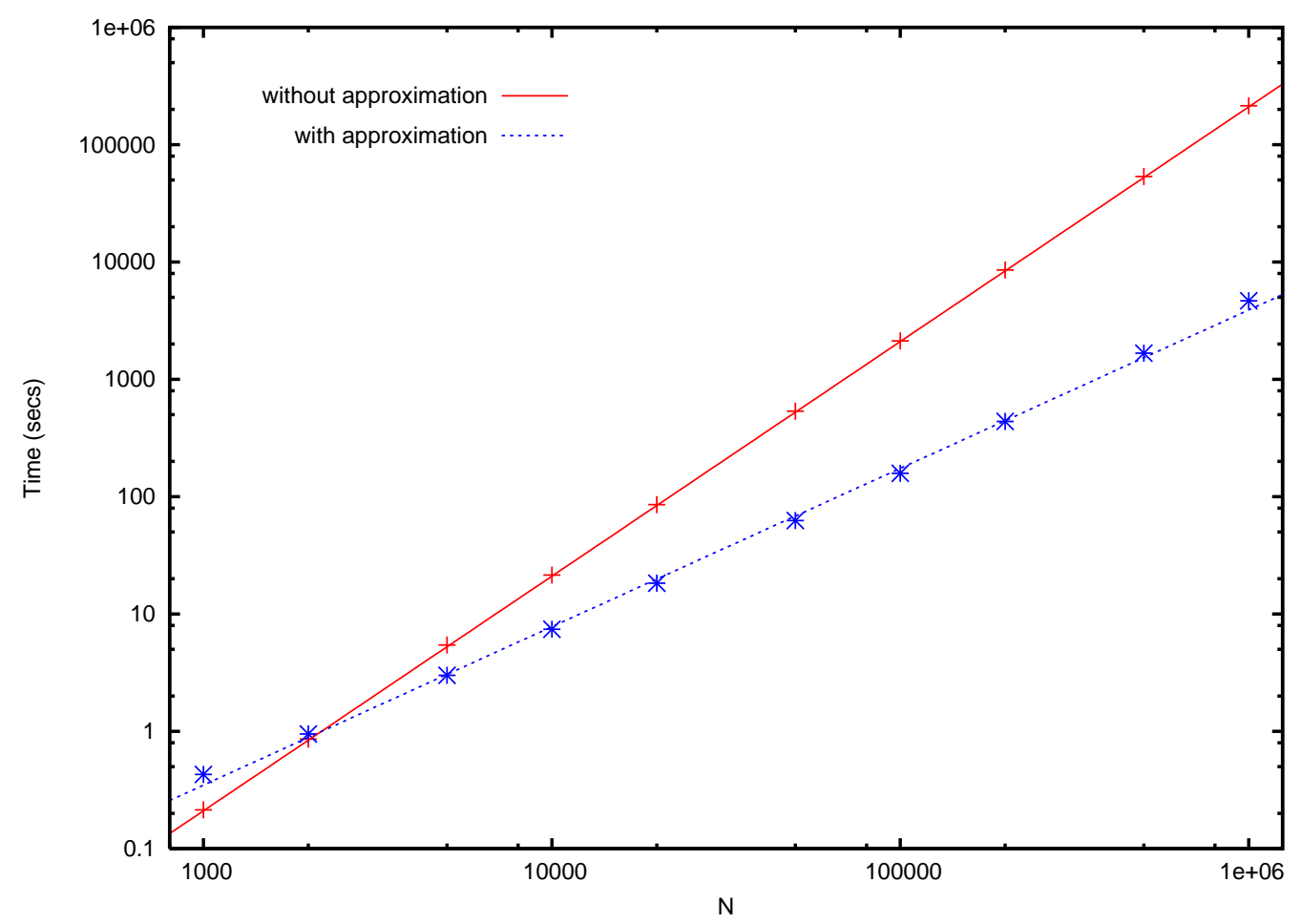

Figure 2: The points are the time (in seconds) needed to unzip a SAW with $N$ steps with and without the power series approximation. The lines have slopes 2 and 1.35.

\section{Comparisons of computational methods}

Given a simple curve, there are several choices when we compute its Loewner driving function. For the explicit conformal map in the zipper algorithm we could use either the map that removes a vertical slit from the half plane or the map that removes a tilted slit. We could use the power series approximation to speed up the calculation as explained in the last section. We have a choice of how many points we use along the curve. In this section we study the effect of these various choices.

For our study we generate a collection of forty self-avoiding walks with one million steps. For each walk we compute its driving function by three different methods. The first uses the vertical slit map and the power series approximation. The second uses the tilted slit map and the power series approximation. The third uses the vertical slit map without the power series approximation. To study the effect of using different numbers of points along the curve we do these computations on subsets of the million points on the SAW. The subsets are obtained by taking every $n$th point along the walk. For the vertical slit map with the power series approximation (the fastest of the three methods) we use $n=1,2,5,10,20,50,100$. So the curves being unzipped have from 1,000,000 to 10,000 points on them. For the tilted slit map with the power series approximation we use $n=2,5,10,20,50,100$. (This method is slower 
than that using vertical slits because of the need to use Newton's method in the computation of the tilted slit conformal map.) For the vertical slit map without the power series approximation (by far the slowest of the three methods) we use $n=5,10,20,50,100$.

Our choice of how to compare the driving functions computed by different methods for the same curve merits some discussion. One might measure the difference between two driving functions by computing the supremum norm or the $L^{1}$ norm of their difference over a bounded interval. We do not do this. The driving functions we are computing are approximations to Brownian motion sample paths. In particular, their slopes can be quite large. If you translate such a function by a small amount, the difference between the translated function and the original function can have a supremum norm or $L^{1}$ norm that is rather large. (Of course, the driving functions are continuous so these norms of the difference go to zero, but not linearly with the size of the translation.) Thus a small error in computing the capacity produces a relatively large error in these norms. Instead of using these norms, we compare driving functions by using only the last point on the driving function. We denote this last point by $\left(T, U_{T}\right)$. The capacity of the SAW is $2 T$ and $U_{T}$ is the image of the end of the SAW under the conformal map that uniformizes the half plane minus the SAW.

We do not know the exact driving function of the SAW, so we treat the result of our computation using all one million steps (i.e., $n=1$ ) with the vertical slit map and the power series approximation as the exact answer. We then compute relative errors for $T$ and $U_{T}$. For $T$ we obtain the relative error by dividing the error in $T$ by $T$. For $U_{T}$ we divide the error by the maximum of $\left|U_{t}\right|$ along the curve. For both of these relative errors we take the average over the forty SAW's.

We begin with the effect of the number of points we use along the curve. We study this using the vertical slit map with the power series approximation. We let $N=10^{6} / n$ denote the number of points on the SAW used in a particular computation. For the vertical slit map with the power series approximation, $N$ ranges from $10^{4}$ to $10^{6}$. In figure 3 we plot the relative error in $T$ as a function of $N$. This is the higher of the two sets of points. In figure 4 we plot the relative error in $U_{T}$ as a function of $N$. Again, this is the higher of the two sets of points. In both of these plots the error is computed by treating the values for $N=10^{6}$ as the exact values. These plots give an idea of the size of the error when the number of points used on the curve is reduced.

To study the effect of the choice of conformal map (vertical slit vs. tilted slit), we compute the difference between the values of $\mathrm{T}$ using the two different maps with the same value of $N$. We do the same for $U_{T}$. We convert these errors to relative errors by dividing by $T$ or the maximum of $\left|U_{t}\right|$. These relative errors are plotted as functions of $N$ in figures 3 and 4 . In both figures they are the lower sets of points. The figure shows they go to zero as $N \rightarrow \infty$. Note that these points lie well below the points that show the difference between the vertical map computation at the given $N$ with the vertical map computation with $N=10^{6}$. In other words, the effect of the choice of the conformal map is smaller than the effect of using more points along the curve.

Finally we discuss the effect of the power series approximation. We use the vertical slit 


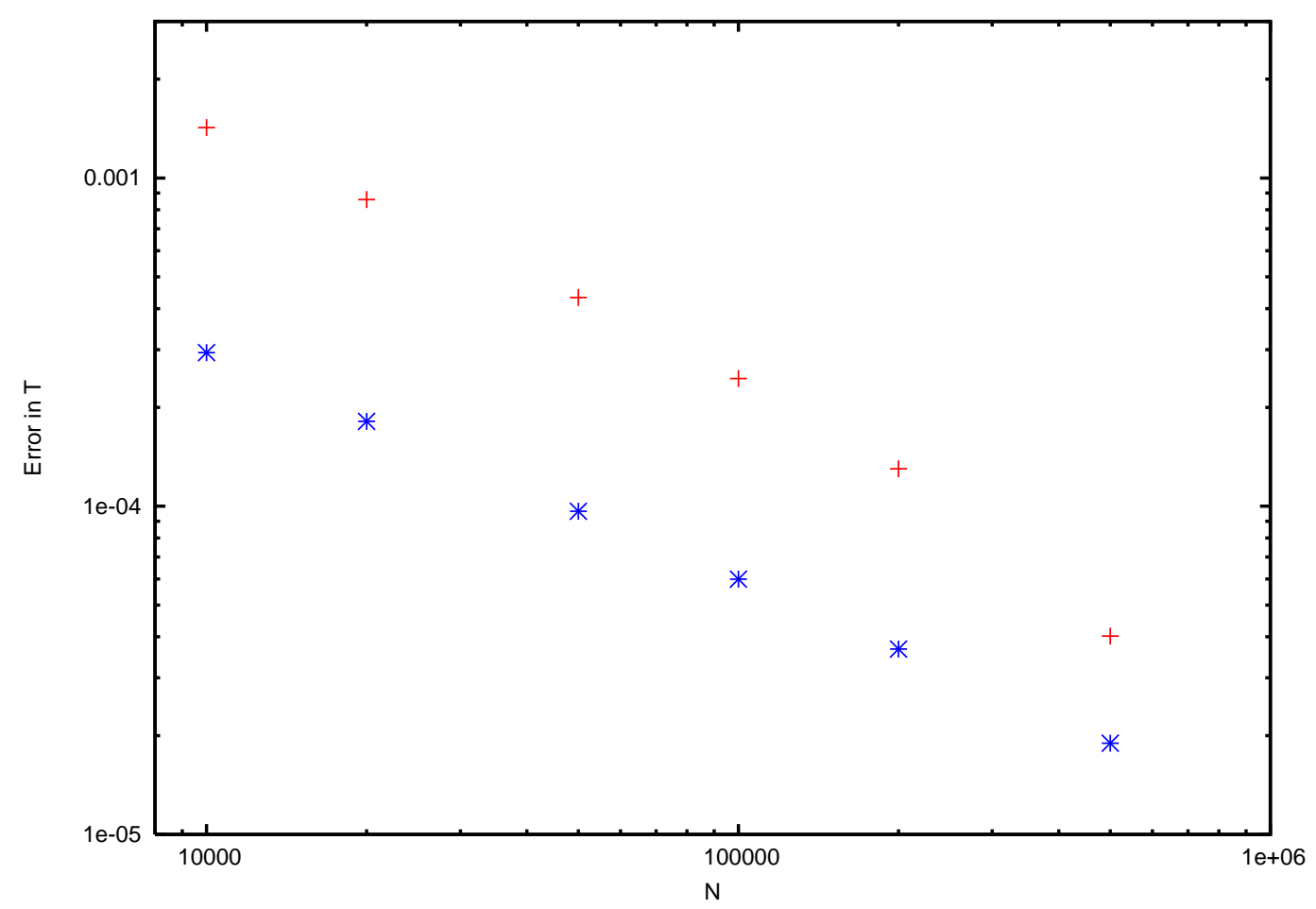

Figure 3: The top set of points is the relative error in $T$ as a function of the number of points $N$ used on the SAW. The bottom set of points is the relative difference between $T$ computed using the tilted slit and vertical slit maps.

\begin{tabular}{|l|c|c|}
\hline $\mathrm{N}$ & $T$ & $U_{T}$ \\
\hline 10,000 & $3.66 \mathrm{e}-09$ & $9.12 \mathrm{e}-09$ \\
20,000 & $2.59 \mathrm{e}-09$ & $7.98 \mathrm{e}-09$ \\
50,000 & $1.61 \mathrm{e}-09$ & $4.73 \mathrm{e}-09$ \\
100,000 & $8.38 \mathrm{e}-10$ & $3.15 \mathrm{e}-09$ \\
200,000 & $6.98 \mathrm{e}-10$ & $1.76 \mathrm{e}-09$ \\
\hline
\end{tabular}

Table 6: The effect of the power series approximation. The table shows the relative differences in $T$ and in $U_{T}$ when we use and do not use the power series approximation.

method. We compute the differences in $T$ and $U_{T}$ computed with and without the power series approximation. We convert these differences to relative differences by dividing by $T$ or the maximum of $\left|U_{t}\right|$. Table 6 shows the relative differences. They are very small and insignificant compared to the differences that we see when we change the conformal map used or the number of points along the curve. In this study we took the number of terms in our power series to be 12 and the parameter $L=4.0$. Increasing either of these improves the accuracy of the 


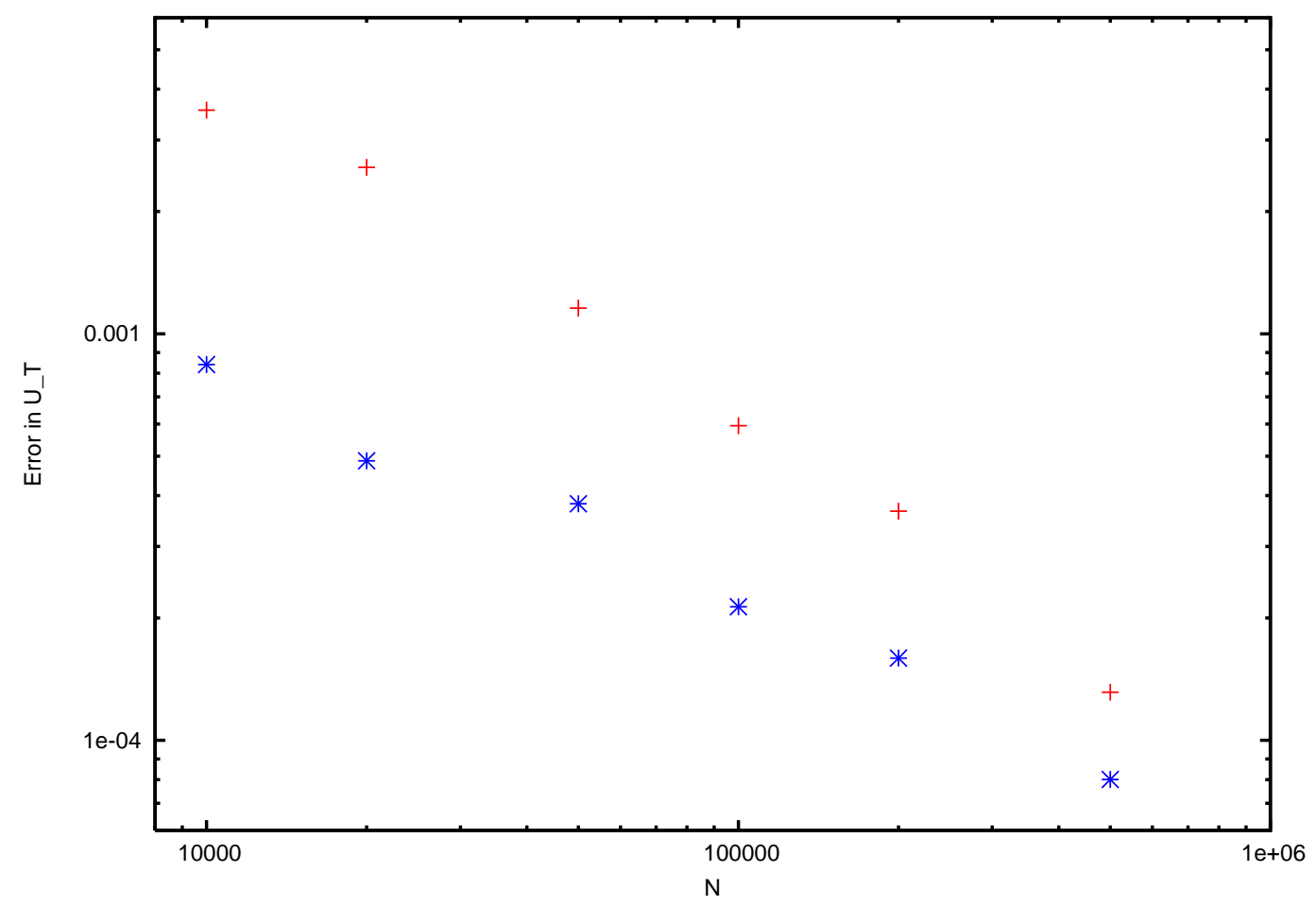

Figure 4: The top set of points is the relative error in $U_{T}$ as a function of the number of points $N$ used on the SAW. The bottom set of points is the relative difference between $U_{T}$ computed using the tilted slit and vertical slit maps.

approximation while slowing down the computation. We used block lengths of 100 .

\section{Conclusions}

In this paper we computed the stochastic driving process of several models of random curves which we know are not SLE. We considered several statistical tests of whether this driving process is a Brownian motion. Simply checking if the distribution at a fixed time is Gaussian was seen to be useless. We must use a test that involves the independence of the increments of the Brownian motion. Our most effective tests were $\chi^{2}$ goodness of fit tests in which we consider $n$ equal increments of the process and take the cells to be determined simply by the signs of the increments. This test was the most successful at concluding that for the models that are not SLE, the stochastic driving process is not a Brownian motion. One nice feature of this test is that it does not involve the value of $\kappa$.

In the models we studied which are not an SLE, we have broken conformal invariance in a drastic way. In particular, these models are not locally isotropic. The models from physics that have been recently studied as possible SLE's should be locally isotropic. So a lack of conformal 
invariance in these physical models would have to arise in a completely different way compared to the models we studied. Our main conclusion is not that the specific tests of Brownian motion that we found effective for our models are the best tests for all models, but rather that when we test for SLE by computing the Loewner driving process, we should employ a variety of tests that this process is a Brownian motion.

We have also studied the numerical problem of computing the driving function of a given curve using the zipper algorithm. We have seen that the difference in the driving function when we use vertical slits or tilted slits for the elementary conformal map for the algorithm is quite small. Given that the vertical slit map is considerably faster and easier to implement, we see no reason to use the tilted slit map. We have also shown that the speed of this algorithm can be increased dramatically using power series approximations of certain analytic functions. The loss of accuracy from this approximation is extremely small, in particular it is insignificant compared to the effect of changing the number of points used to define the curve we are unzipping or compared to the difference between using vertical slits or tilted slits in the algorithm.

\section{A Details of the simulations}

In this appendix we give some details of the simulations of the distorted LERW, SAW and percolation interface.

The LERW walk that we simulate is chordal LERW in the half plane from 0 to $\infty$. This means that we take an ordinary random walk beginning at the origin and condition it to remain in the upper half plane. Then we erase the loops in chronological order. The ordinary random walk conditioned to remain in the upper half plane is easy to simulate since it is given by a random walk beginning at 0 with transition probabilities that only depend on the vertical component of the present location of the walk. If the site has vertical component $k$, then the walk moves up with probability $(k+1) / 4 k$, down with probability $(k-1) / 4 k$, and to the right or left with probability $1 / 4$. (See, for example, section 0.1 of [9]). This process is known as the half plane excursion. The half-plane excursion is transient, i.e., each lattice site is visited by the excursion a finite number of times. This implies that the loop erasure makes sense. (For a transient walk all parts of the walk would eventually be part of a loop and so would be erased.) Note, however, that if we take an infinite half plane excursion and only consider the first $n$ steps and loop-erase this walk, the result does not completely agree with the loop-erasure of the full infinite excursion. A site which is visited by the excursion before time $n$ may be erased by a loop formed after time $n$.

In practice there is no way to know if a visit to a site will be erased by some future loop without simulating the entire excursion. So in the simulation we do the following. We generate a half-plane excursion, erasing the loops as they are formed. We stop when the resulting walk has $N$ steps. If $n$ is small compared to $N$, then the distribution of our walk for the first $n$ steps is close to the true distribution of the first $n$ steps of the LERW. We will only compute the driving function for the first $n$ steps. 
We take $N=50,000$ and generate 100,000 samples. If we work on a unit lattice, a LERW with $N$ steps has a size of order $N^{\nu}$ with $\nu=4 / 5$. So we rescale our walk by a factor of $N^{\nu}$ to obtain a curve whose size is of order one. We then compute its driving function up to time $T=0.01$. The time $T$ is one half of the capacity. So the number of steps needed to reach $T=0.01$ is random. For this choice of $T$, the mean of this random number of steps is approximately 8200, roughly a factor of six smaller than $N$.

The SAW in the upper half plane is defined as follows. Let $N$ be a positive integer. We consider all nearest neighbor walks with $N$ steps in the upper half plane which begin at the origin and do not visit any site more than once. We put the uniform probability measure on this finite set of walks. We let $N \rightarrow \infty$ to get a probability measure on infinite self-avoiding walks on the unit lattice in the upper half plane. Then we take the lattice spacing to zero. We simulate the SAW in the half plane with a fixed number of steps with the pivot algorithm, a Markov Chain Monte Carlo method [12]. We use the fast implementation of this algorithm introduced in [7]. For the SAW there is an issue similar to the LERW. The pivot algorithm produces the uniform distribution on the set of walks with $N$ steps. But this is not the distribution of the infinite SAW in the half plane restricted to walks of length $N$. As with the LERW, we address this problem by simulating walks with $N$ steps but then computing the driving function for only the first $n$ steps where $n$ is much smaller than $N$.

We simulate SAW's with 200,000 steps. We sample the SAW from the pivot algorithm every 100, 000 time steps in the Markov chain. We run the chain for $10^{10}$ iterations to produce 100, 000 samples. Unlike the other two models, these samples are not exactly independent, but the large time interval between sampling makes the samples very close to independent. We rescale the SAW by a factor of $N^{\nu}$ with $\nu=3 / 4$ and then compute its driving function up to time $T=0.002$. The mean of the number of steps needed to reach $T=0.002$ is approximately 9350, roughly a factor of 20 smaller than the total number of steps in the SAW.

The percolation model we study is site percolation on the triangular lattice in the upper half plane, but we describe it using the hexagonal lattice in the upper half plane. Each hexagon is colored white or black with probability $1 / 2$. The hexagons along the negative real axis are white and those along the positive real axis are black. This forces an interface which starts with the bond through the origin between the adjacent differently colored hexagons on the real axis. This interface is the unique curve on the hexagonal lattice which begins at this bond and has all white hexagons along one side of the interface and all black ones along the other side.

Note that unlike the LERW or SAW there is no finite length effect for percolation interfaces. If we generate interfaces with $n$ steps, they have exactly the same distribution as the first $n$ steps of interfaces of length $N$ where $N>n$. We generate 100,000 samples of interfaces with $N=40,000$ steps. We rescale our walk by a factor of $N^{\nu}$ with $\nu=4 / 7$ and then compute the driving function up to time $T=0.1$. This corresponds to a mean number of steps of approximately 11,300 .

We end with a comment on the time $T$ and our rescaling of the various curves. For each model we have rescaled the curves by a factor of $N^{\nu}$. This is merely for convenience. We could have left the curves on a unit lattice and computed the driving function up to time $T$ given 
by the above values times $N^{2 \nu}$. What is important is that the mean number of steps of the curves we are unzipping is large (so that we are close to the scaling limit) but still significantly smaller than the total number of steps in the curve (for the SAW and LERW) so that we avoid the finite length effects discussed above. We have chosen the values of $T$ so that in all three models the mean number of steps unzipped is on the order of 10,000. Almost all of the time in these simulations is spent on computing the driving functions. Generating the random curves takes essentially no time by comparison.

Acknowledgments: This research was inspired by talks and interactions during a visit to the Kavli Institute for Theoretical Physics in September, 2006. I thank Don Marshall and Stephen Rohde for useful discussions about the zipper algorithm. This research was supported in part by the National Science Foundation under grant DMS-0501168.

\section{References}

[1] C. Amoruso, A. K. Hartman, M. B. Hastings, and M. A. Moore, Conformal invariance and SLE in two-dimensional Ising spin glasses, Phys. Rev. Lett. 97, 267202 (2006). Archived as arXiv: cond-mat/0601711.

[2] D. Bernard, G. Boffetta, A. Celani, and G. Falkovich, Conformal invariance in two-dimensional turbulence, Nature Physics 2, 124 (2006). Archived as arXiv:nlin.CD/0602017.

[3] D. Bernard, G. Boffetta, A. Celani, and G. Falkovich, Inverse turbulent cascades and conformally invariant curves. Archived as arXiv:nlin.CD/0609069.

[4] D. Bernard, P. Le Doussal, and A. A. Middleton, Are domain walls in 2D spin glasses described by stochastic Loewner evolutions?, Phys. Rev. B 76, 020403(R) (2007). Archived as arXiv: cond-mat/0611433.

[5] F. Camia and C. M. Newman, Critical percolation exploration path and SLE(6): a proof of convergence, preprint. Archived as arXiv:math.PR/0604487.

[6] T. Kennedy, A fast algorithm for simulating the chordal Schramm-Loewner evolution, $J$. Statist. Phys. 128, 1125-1137 (2007). Archived as arXiv:math.PR/0508002.

[7] T. Kennedy, A faster implementation of the pivot algorithm for self-avoiding walks, $J$. Statist. Phys. 106, 407-429 (2002). Archived as arXiv:cond-mat/0109308 
[8] R. Kühnau, Numerische Realisierung konformer Abbildungen durch "Interpolation", $Z$. Angew. Math. Mech. 63, 631-637 (1983).

[9] G. Lawler, Conformally Invariant Processes in the Plane, Mathematical Surveys and Monographs, vol. 114, American Mathematical Society, 2005.

[10] G. Lawler, O. Schramm, and W.Werner, On the scaling limit of planar self-avoiding walk, Fractal Geometry and Applications: a Jubilee of Benoit Mandelbrot, Part 2, 339364, Proc. Sympos. Pure Math. 72, Amer. Math. Soc., Providence, RI, 2004. Archived as arXiv:math.PR/0204277.

[11] G. Lawler, O. Schramm, and W.Werner, Conformal invariance of planar loop-erased random walks and uniform spanning trees, Ann. Probab. 32, 939-995 (2004). Archived as arXiv:math.PR/0112234.

[12] N. Madras and G. Slade, The Self-Avoiding Walk, Birkhäuser, Boston-Basel-Berlin, 1993.

[13] D. E. Marshall and S. Rohde, Convergence of a variant of the Zipper algorithm for conformal mapping, SIAM J. Numer. Anal. to appear.

[14] O. Schramm, Scaling limits of loop-erased random walks and uniform spanning trees, Israel J. Math. 118, 221-288 (2000). Archived as arXiv:math.PR/9904022.

[15] S. Smirnov, Critical percolation in the plane, C. R. Acad. Sci. Paris Sér. I Math. 333, 239 (2001).

[16] S. Smirnov, Towards conformal invariance of 2D lattice models, Proceedings of the International Congress of Mathematicians, Vol. II, 1421-1451, Eur. Math. Soc., Zurich, 2006. Archived as arXiv:0708.0032v1 [math-ph].

[17] O. Schramm and S. Sheffield, Contour lines of the two-dimensional discrete Gaussian free field, preprint. Archived as arXiv:math.PR/0605337.

[18] J. Tsai, The Loewner driving function of trajectory arcs of quadratic differentials, preprint. Archived as arXiv:0704.1933v2 [math.CV].

[19] D. Zhan, The scaling limits of planar LERW in finitely connected domains, Ann. Probab. 36, 467-529 (2008). Archived as arXiv:math.PR/0610304. 